\title{
ÜBER PERIODISCHE LÖSUNGEN KOERZITIVER LINEARER PARTIELLER DIFFERENTIALGLEICHUNGEN
}

\author{
ILPPO SIMO LOUHIVAARA und CHRISTIAN G. SIMADER
}

Herrn Professor Dr. Dr. h. c. Béla Szökefalvi-Nagy in Verehrung gewidmet

\section{Einführung}

0.1. In der vorliegenden Note betrachten wir Klassen linearer partieller Differentialoperatoren, die auf periodische Funktionen wirken. Diese Klassen sind durch Koerzitivitätsungleichungen, z. B. vom Typ der Gårdingschen Ungleichung, gekennzeichnet. Wir geben zunächst eine algebraische Charakterisierung dieser Klassen für Differentialoperatoren mit konstanten Koeffizienten an. Aus dieser algebraischen Charakterisierung ist ersichtlich, daß den von uns betrachteten Klassen von Differentialoperatoren auch nichthypoelliptische Operatoren angehören (man vergleiche [10]); dies macht es angesichts der fundamentalen Resultate von Lars Hörmander [5] und [6], S. 96-114, klar, daß Regularitätsaussagen über die im geeigneten Sinne schwachen Lösungen (wie auch in [10]) nur global und auch nur unter gewissen einschränkenden Bedingungen gewonnen werden können.

Im Falle der Differentialoperatoren mit konstanten Koeffizienten erschließen wir aus den Koerzitivitätsungleichungen die Existenz und Regularität schwacher und starker periodischer Lösungen der zugehörigen Differentialgleichungen. Diese Resultate übertragen sich auf solche Differentialoperatoren mit variablen periodischen Koeffizienten, die in geeignetem Sinne wenig von je einem koerzitiven Differentialoperator mit konstanten Koeffizienten abweichen.

Im Falle elliptischer Differentialoperatoren sind Regularitätsresultate für periodische Lösungen benutzt worden, um die Regularität schwacher Lösungen elliptischer Randwertprobleme zu zeigen (man vergleiche Peter D. Lax [8] sowie auch Agmon [1], S. 26-61, Bers-Schechter [2], S. 164-210, Eells [3], S. 105-124, und Hildebrandt [4], S. 59-89, 147-200). In gleicher Weise ist es wohl möglich, die hier erzielten Resultate zum Beweis der inneren Regularität schwacher Lösungen von Randwertproblemen für hypoelliptische koerzitive Differentialoperatoren heranzuziehen. 
0.2. Es sei

$$
W:=\left\{x=\left(x_{1}, \ldots, x_{n}\right) \in \boldsymbol{R}^{n} \mid-\pi<x_{j}<\pi, j=1, \ldots, n\right\}
$$

der Periodenwürfel in $\left.\boldsymbol{R}^{n}(n \in N)^{1}\right)$ und $\bar{W}$ seine Abschließung. Für geordnete Systeme $x=\left(x_{1}, \ldots, x_{n}\right) \quad\left(\in \boldsymbol{R}^{n}\right)$ und $\xi=\left(\xi_{1}, \ldots, \xi_{n}\right) \quad\left(\in \boldsymbol{R}^{n}\right)$ von je $n$ reellen Zahlen schreiben wir $(x, \xi):=x_{1} \xi_{1}+\ldots+x_{n} \xi_{n}$ und $|x|:=\left(x_{1}^{2}+\ldots+x_{n}^{2}\right)^{1 / 2}$. Für einen Multiindex $\sigma$, d.h. für ein geordnetes System $\sigma:=\left(\sigma_{1}, \ldots, \sigma_{n}\right) \quad\left(\in N_{0}^{n}\right)$ von $n$ nichtnegativen ganzen Zahlen sei $|\sigma|:=\sigma_{1}+\ldots+\sigma_{n}$ und $\sigma !:=\sigma_{1} ! \ldots \sigma_{n} !$. Ferner sei $\xi^{\sigma}:=\xi_{1}^{\sigma_{1}} \ldots \xi_{n}^{\sigma_{n}}$ für $\xi=\left(\xi_{1}, \ldots, \xi_{n}\right) \in \boldsymbol{R}^{n}$ und $D^{\sigma}:=D_{1}^{\sigma_{1}} \ldots D_{n}^{\sigma_{n}}$ mit $D:=\left(D_{1}, \ldots, D_{n}\right)$ und $D_{j}:=-i \partial / \partial x_{j}$.

Es sei $S$ die Menge der Funktionen $\varphi$ aus $C^{\infty}(\bar{W})$, die als endliche Summen

$$
\varphi(x)=(2 \pi)^{-n / 2} \sum_{k \in Z^{n}} \varphi_{k} e^{i(k, x)}
$$

mit Koeffizienten $\varphi_{k} \in \boldsymbol{C}$ darstellbar sind, d.h. zu jedem $\varphi$ sind die Koeffizienten $\varphi_{k}$ gleich Null außer für eine endliche Menge von $k \in \boldsymbol{Z}^{n}$.

Für komplexwertige Funktionen $f, g \in L^{2}(W)$ schreiben wir

und $\|f\|:=(f, f)^{1 / 2}$.

$$
(f, g):=\int_{W} \overline{f(x)} g(x) d x
$$

Nach der Parsevalschen Gleichung folgt für $\varphi, \psi \in S$

mit

$$
(\varphi, \psi)=\sum_{k \in Z^{n}} \bar{\varphi}_{k} \psi_{k}
$$

$$
\varphi_{k}:=(2 \pi)^{-n / 2} \int_{W} \varphi(x) e^{-i(k, x)} d x .
$$

Für $s \in \boldsymbol{R}$ und $\varphi, \psi \in S$ setzen wir

$$
(\varphi, \psi)_{s}:=\sum_{k \in Z^{n}}\left(1+|k|^{2}\right)^{s} \bar{\varphi}_{k} \psi_{k}
$$

und $\|\varphi\|_{s}:=(\varphi, \varphi)_{s}^{1 / 2}$. Es gilt $(\varphi, \psi)_{0}=(\varphi, \psi)$ für alle $\varphi, \psi \in S$.

Mit $H_{\pi}^{s}(s \in \boldsymbol{R})$ bezeichnen wir die Vervollständigung von $S$ bezüglich des durch $(0.1)$ definierten Skalarproduktes $(,)_{s}$. Die Elemente $u$ des Raumes $H_{\pi}^{s}$ können identifiziert werden mit den Zahlenfolgen $\left\{u_{k}\right\}_{k \in Z^{n}}$, für die

$$
\sum_{k \in Z^{n}}\left(1+|k|^{2}\right)^{s}\left|u_{k}\right|^{2}<\infty
$$

gilt. Für $u \in H_{\pi}^{s}$ schreiben wir somit auch $u=\left\{u_{k}\right\}_{k \in Z^{n}}$.

Mit $s \in N$ gilt

$$
\left((1-\Delta)^{s} \varphi, \psi\right)=(\varphi, \psi)_{s} \text { für alle } \varphi, \psi \in S,
$$

wobei $\Delta$ den Laplaceoperator bezeichnet.

1) Wir bezeichnen mit $\boldsymbol{R}$ den Körper der reellen Zahlen, mit $\boldsymbol{C}$ den Körper der komplexen Zahlen, mit $Z(\subset R)$ die Menge der ganzen Zahlen, mit $N(\subset Z)$ die Menge der positiven ganzen Zahlen und mit $N_{0}$ die Menge $N \cup\{0\}$. 
Die Räume $H_{\pi}^{s}$ und $H_{\pi}^{-s}(s \in \boldsymbol{R})$ sind dual bezüglich der Form (, ). Für $s \in N$ ist $H_{\pi}^{s}$ isometrisch isomorph dem Raum der mit dem Periodenwürfel $W$ (d.h. in allen Variablen mit Periode $2 \pi$ ) periodischen Funktionen aus dem üblichen Sobolevraum $H^{s}(W)$ (für dieses und für weitere Eigenschaften der Räume $H_{\pi}^{s}$ sei auf Bers-Schechter [2], S. 165-170, und Eells [3], S. 105-112, verwiesen).

Mit $C_{\pi}^{r}$ bezeichnen wir die Menge der mit dem Periodenwürfel $W$ periodischen komplexwertigen Funktionen aus $C^{r}(\bar{W})$, und es sei $C_{\pi}^{\infty}:=\bigcap_{r=0}^{\infty} C_{\pi}^{r}$.

\section{Koerzitive Differentialoperatoren. Lösungsbegriffe}

1.1. Wir betrachten einen linearen partiellen Differentialoperator

$$
L(x, D):=\sum_{|\sigma| \leqq r} a_{\sigma}(x) D^{\sigma}
$$

mit Koeffizienten $a_{\sigma} \in C_{\pi}^{|\sigma|}$.

Der dem Differentialoperator $L(., D)$ formal adjungierte Differentialoperator $L^{\prime}(., D)$ ist durch

erklärt, und es gilt

$$
L^{\prime}(x, D) u(x):=\sum_{|\sigma| \leqq r} D^{\sigma}\left(\overline{a_{\sigma}(x)} u(x)\right)
$$

$$
(L(., D) u, v)=\left(u, L^{\prime}(., D) v\right) \quad \text { für alle } u, v \in C_{\pi}^{r} .
$$

Dem Differentialoperator $L(., D)$ ordnen wir durch Substitution $D \mapsto \xi \in \boldsymbol{R}^{n}$ das Polynom

der Veränderlichen $\xi$ zu.

$$
L(x, \xi):=\sum_{|\sigma| \leqq r} a_{\sigma}(x) \xi^{\sigma}
$$

Definition 1.1. Der Differentialoperator $L(., D)$ heißt $t$-koerzitiv bzw. stark $t$-koerzitiv $(t \in \boldsymbol{R}, t>0)$, falls zwei Konstanten $C_{1}>0$ und $C_{2} \geqq 0$ so existieren, daß

$$
\|L(., D) \varphi\| \geqq C_{1}\|\varphi\|_{t}-C_{2}\|\varphi\| \quad \text { für alle } \varphi \in S
$$

bzw.

$$
\operatorname{Re}(L(., D) \varphi, \varphi) \geqq C_{1}\|\varphi\|_{t / 2}^{2}-C_{2}\|\varphi\|^{2} \quad \text { für alle } \varphi \in S
$$

gilt.

Im Falle der konstanten Koeffizienten besteht folgende Charakterisierung:

Satz 1.2. Damit der Differentialoperator

$$
L(D):=\sum_{|\sigma| \leqq r} a_{\sigma} D^{\sigma}
$$


mit konstanten Koeffizienten $a_{\sigma} \in \boldsymbol{C}$ t-koerzitiv bzw. stark $t$-koerzitiv ist, ist notwendig und hinreichend, daß zwei Konstanten $E>0$ und $R \geqq 0$ existieren, mit denen

$$
\left|\sum_{|\sigma| \leqq r} a_{\sigma} k^{\sigma}\right| \geqq E|k|^{t} \quad \text { für alle } k \in \boldsymbol{Z}^{n} \text { mit }|k| \geqq R
$$

bzw.

$$
\operatorname{Re} \sum_{|\sigma| \leqq r} a_{\sigma} k^{\sigma} \geqq E|k|^{t} \quad \text { für alle } k \in Z^{n} \text { mit }|k| \geqq R
$$

gilt.

Folglich ist ein stark $t$-koerzitiver Differentialoperator mit konstanten Koeffizienten auch $t$-koerzitiv.

Beweis. Wir werden den Fall eines stark $t$-koerzitiven Differentialoperators hier behandeln, der Beweis im Falle eines $t$-koerzitiven Differentialoperators verläuft analog.

A. Sei $L(D)$ stark $t$-koerzitiv. Man setze in Ungleichung (1.2b) die durch

$$
\varphi(x):=(2 \pi)^{-n / 2} e^{i(k, x)}
$$

mit $k \in \boldsymbol{Z}^{n}$ erklärte Funktion $\varphi \in S$ ein. Dann ist

$$
L(D) \varphi(x)=(2 \pi)^{-n / 2} L(k) e^{i(k, x)},
$$

und daher folgt aus (1.2b)

$$
\operatorname{Re} \overline{L(k)} \geqq C_{1}\left(1+|k|^{2}\right)^{t / 2}-C_{2} .
$$

Mit

ergibt sich (1.4b).

$$
R:=\left(2 C_{2} / C_{1}\right)^{1 / t} \text { und } E:=C_{1} / 2
$$

B. Der Differentialoperator $L(D)$ erfülle Bedingung (1.4b) mit $E>0$ und $R \geqq 0$. Für $k \in \boldsymbol{Z}^{n}$ mit $|k| \geqq 1$ ist

$$
\left(1+|k|^{2}\right)^{t / 2} \leqq 2^{t / 2}|k|^{t}
$$

Also ist für $|k| \geqq R_{0}:=\max \{1, R\}$

$$
\operatorname{Re} L(k) \geqq C_{1}\left(1+|k|^{2}\right)^{t / 2}
$$

mit $C_{1}:=E / 2^{t / 2}$. Weiter gilt mit einer Konstanten $C_{3}>0$ die Ungleichung

Für jedes $\varphi \in S$,

$$
|L(k)| \leqq C_{3}\left(1+|k|^{2}\right)^{r / 2} .
$$

$$
\varphi(x):=(2 \pi)^{-n / 2} \sum_{k \in Z^{n}} \varphi_{k} e^{i(k, x)}
$$


ergibt sich

$$
\begin{aligned}
& \operatorname{Re}(L(D) \varphi, \varphi)=\sum_{k \in Z^{n}}\left|\varphi_{k}\right|^{2} \operatorname{Re} L(k) \\
= & \sum_{|k| \geqq R_{0}}\left|\varphi_{k}\right|^{2} \operatorname{Re} L(k)+\sum_{|k|<R_{0}}\left|\varphi_{k}\right|^{2} \operatorname{Re} L(k) \\
\geqq & C_{1} \sum_{|k| \leqq R_{0}}\left|\varphi_{k}\right|^{2}\left(1+|k|^{2}\right)^{t / 2}-C_{3} \sum_{|k|<R_{0}}\left|\varphi_{k}\right|^{2}\left(1+|k|^{2}\right)^{r / 2} \\
\geqq & C_{1} \sum_{k \in Z^{n}}\left|\varphi_{k}\right|^{2}\left(1+|k|^{2}\right)^{t / 2} \\
& -\sum_{|k|<R_{0}}\left|\varphi_{k}\right|^{2}\left(C_{1}\left(1+|k|^{2}\right)^{t / 2}+C_{3}\left(1+|k|^{2}\right)^{r / 2}\right) \\
\geqq & C_{1}\|\varphi\|_{t / 2}^{2}-C_{2}\|\varphi\|^{2}
\end{aligned}
$$

mit $C_{2}:=C_{1}\left(1+R_{0}^{2}\right)^{t / 2}+C_{3}\left(1+R_{0}^{2}\right)^{r / 2}$.

1.2. Wie es bei der Behandlung von Differentialgleichungsproblemen mit Hilfe von Hilbertraummethoden üblich ist, ordnen wir den Differentialoperatoren $L(., D)$ und $L(D)$ gewisse lineare Operatoren in geeigneten Hilberträumen zu. (Koerzivität dieser Operatoren wird hierbei nicht vorausgesetzt.)

A. Sei $L(., D)$ der Differentialoperator (1.1) mit Koeffizienten $a_{\sigma} \in C_{\pi}^{|\sigma|}$. Zu dem diesem Differentialoperator $L(., D)$ formal adjungierten Differentialoperator $L^{\prime}(., D)$ ist in $H_{\pi}^{0}$ durch

$$
\begin{gathered}
D\left(L^{\prime}\right):=S \subset H_{\pi}^{0}, \\
L^{\prime} \varphi:=L^{\prime}(., D) \varphi \text { für } \varphi \in S
\end{gathered}
$$

ein linearer Operator $L^{\prime}: D\left(L^{\prime}\right) \rightarrow H_{\pi}^{0}$ mit der in $H_{\pi}^{0}$ dichten Definitionsmenge $D\left(L^{\prime}\right)$ erklärt.

Weil

$$
\left(\psi, L^{\prime} \varphi\right)_{0}=\left(\psi, L^{\prime}(., D) \varphi\right)=(L(., D) \psi, \varphi) \text { für alle } \varphi, \psi \in S
$$

gilt, ist $S \subset D\left(L^{\prime *}\right)$ und $L^{* *} \psi=L(., D) \psi$ für alle $\psi \in S$ (mit * wird der bezüglich des betreffenden Hilbertraumskalarproduktes adjungierte Operator bezeichnet). Daher ist $D\left(L^{\prime *}\right)$ dicht in $H_{\pi}^{0}$, und $L^{\prime}$ ist deshalb abschließbar mit der kleinsten abgeschlossenen Fortsetzung $L^{\prime \sim}:=L^{\prime * *}$.

Sei $f \in H_{\pi}^{0}$ vorgegeben. Unter einer schwachen Lösung (einer schwachen $H_{\pi}^{0}$ Lösung) der Differentialgleichung

$$
L(., D) u=f
$$

verstehen wir jedes $u \in D\left(L^{\prime *}\right)$ mit $L^{\prime *} u=f$.

Eine äquivalente Charakterisierung der schwachen Lösung ist enthalten in der Forderung für $u \in H_{\pi}^{0} \subset L^{2}(W)$, daß

gilt.

$$
\left(u, L^{\prime}(., D) \varphi\right)=(f, \varphi) \text { für alle } \varphi \in S
$$


Ist $u$ eine schwache Lösung der Differentialgleichung (1.5) und gilt darüberhinaus $u \in C_{\pi}^{r}$ und $f \in C_{\pi}^{0}$, so ist $u$ auch im klassischen Sinne eine (mit dem Periodenwürfel $W$ ) periodische Lösung der Differentialgleichung (1.5).

B. Sei dann speziell $L(D)$ ein Differentialoperator (1.3) mit konstanten Koeffizienten $a_{\sigma} \in \boldsymbol{C}$. Dann läßt sich der eben eingeführte Begriff der schwachen Lösung auf $H_{\pi}^{s}$ mit beliebigem $s \in \boldsymbol{R}$ (statt $H_{\pi}^{0}$ ) übertragen:

Sei $f \in H_{\pi}^{s}$ vorgegeben. Unter einer schwachen $H_{\pi}^{s}$-Lösung der Differentialgleichung

$$
L(D) u=f
$$

verstehen wir jedes $u \in H_{\pi}^{s}$ mit

$$
\left(u, L^{\prime}(D) \varphi\right)_{s}=(f, \varphi)_{s} \quad \text { für alle } \varphi \in S .
$$

C. Sei wieder $L(., D)$ der Differentialoperator (1.1) mit variablen, periodischen und genügend oft stetig differenzierbaren Koeffizienten $a_{\sigma}$; der Einfachheit halber nehme man an dieser Stelle $a_{\sigma} \in C_{\pi}^{\infty}$ an. In $H_{\pi}^{s}$ mit $s \in \boldsymbol{R}$ ist durch

$$
\begin{gathered}
D\left(L_{s}\right):=S \subset H_{\pi}^{s}, \\
L_{s} \varphi:=L(., D) \varphi \quad \text { für } \varphi \in S
\end{gathered}
$$

ein (in $H_{\pi}^{s}$ dicht definierter) abschließbarer linearer Operator $L_{s}: D\left(L_{s}\right) \rightarrow H_{\pi}^{s}$ mit der kleinsten abgeschlossenen Fortsetzung $L_{s}^{\sim}:=L_{s}^{* *}$ erklärt. Wir schreiben $L:=L_{0}$ und $L^{\sim}:=L_{0}^{\sim}$.

Mit vorgegebenem $f \in H_{\pi}^{s}$ wird jedes $u \in D\left(L_{s}^{\sim}\right)$ mit $L_{s}^{\sim} u=f$ eine starke $H_{\pi}^{s}$-Lösung der Differentialgleichung (1.5) genannt.

Jede starke $H_{\pi}^{0}$-Lösung der Differentialgleichung (1.5) ist auch eine schwache $H_{\pi}^{0}$-Lösung; die Umkehrung braucht allgemein nicht zu gelten. In dem Falle, daß $L^{\sim}=L^{* *}$ gilt, heißt $L$ wesentlich maximal.

\section{Der Fall konstanter Koeffizienten}

2.1. Zunächst betrachten wir lineare Differentialoperatoren mit konstanten Koeffizienten, sogar ohne Koerzivität vorauszusetzen, und beweisen:

Satz 2.1. Sei

$$
L(D):=\sum_{|\sigma| \leqq r} a_{\sigma} D^{\sigma}
$$

ein Differentialoperator mit konstanten Koeffizienten $a_{\sigma} \in \boldsymbol{C}$, und sei $s \in \boldsymbol{R}$.

A. Sei $f=\left\{f_{k}\right\}_{k \in Z^{n}} \in H_{\pi}^{s}$, und sei $u=\left\{u_{k}\right\}_{k \in Z^{n}} \in H_{\pi}^{s}$ eine schwache $H_{\pi}^{s}$-Lösung der Differentialgleichung

d.h. $u$ genügt der Gleichung

$$
L(D) u=f
$$

$$
\left(u, L^{\prime}(D) \varphi\right)_{s}=(f, \varphi)_{s} \text { für alle } \varphi \in S .
$$


Dann gilt

$$
L(k) u_{k}=f_{k} \quad \text { für alle } k \in \boldsymbol{Z}^{n} .
$$

B. Relation (2.4) gelte für $f=\left\{f_{k}\right\}_{k \in Z^{n}} \in H_{\pi}^{s}$ und $u=\left\{u_{k}\right\}_{k \in Z^{n}} \in H_{\pi}^{s}$. Dann gilt $L_{s}^{\sim} u=f, d . h . u$ ist eine starke $H_{\pi}^{s}$-Lösung der Differentialgleichung (2.2).

C. Für die Nullräume $N\left(L_{s}^{\sim}\right)$ und $N\left(L_{s}^{*}\right)$ sowie für die Wertemengen $R\left(L_{s}^{\sim}\right)$ und $R\left(L_{s}^{*}\right)$ von $L_{s}^{\sim}$ bzw. $L_{s}^{*}$ gelten die Beziehungen

$$
N\left(L_{s}^{\tilde{s}}\right)=N\left(L_{s}^{*}\right), \quad R\left(L_{s}^{\sim}\right)=R\left(L_{s}^{*}\right) .
$$

D. Die Definitionsmenge $D\left(L_{s}^{\sim}\right)$ von $L_{s}^{\sim}$ fällt mit der Menge derjenigen Elemente $u=\left\{u_{k}\right\}_{k \in Z^{n}} \in H_{\pi}^{s}$ zusammen, für die

$$
\sum_{k \in Z^{n}}\left(1+|k|^{2}\right)^{s}\left|u_{k}\right|^{2}|L(k)|^{2}<\infty
$$

gilt.

E. Ein $u=\left\{u_{k}\right\}_{k \in Z^{n}} \in H_{\pi}^{s}$ gehört zu $N\left(L_{s}^{\sim}\right)$ genau dann, wenn $u_{k}=0$ für alle $k \in \boldsymbol{Z}^{n}$ mit $L(k) \neq 0$ gilt.

F. Ferner besteht die Wertemenge $R\left(L_{s}^{\sim}\right)$ von $L_{s}^{\sim}$ genau aus denjenigen $f=\left\{f_{k}\right\}_{k \in Z^{n}} \in H_{\pi}^{s}$, für die durch

$$
u_{k}:= \begin{cases}f_{k} / L(k) & \text { für } L(k) \neq 0 \\ 0 & \text { für } L(k)=0\end{cases}
$$

je ein Element $u=\left\{u_{k}\right\}_{k \in Z^{n}}$ in $H_{\pi}^{s}$ erklärt ist.

Beweis. A. Aus (2.3) folgt für

$$
\varphi(x):=(2 \pi)^{-n / 2}\left(1+|k|^{2}\right)^{-s / 2} e^{i(k, x)}
$$

mit $k \in \boldsymbol{Z}^{\boldsymbol{n}}$ Behauptung (2.4).

B. Definiert man für $j \in N$

so gilt

$$
\varphi_{j}(x):=(2 \pi)^{-n / 2} \sum_{|k| \leqq j} u_{k} e^{i(k, x)},
$$

Ferner ist

und aus (2.4) folgt

$$
\left\|u-\varphi_{j}\right\|_{s} \rightarrow 0 \text { für } j \rightarrow \infty \text {. }
$$

$$
L(D) \varphi_{j}(x)=(2 \pi)^{-n / 2} \sum_{|k| \leqq j} L(k) u_{k} e^{i(k, x)},
$$

somit gilt

$$
\sum_{|k| \leqq j}\left(1+|k|^{2}\right)^{s}\left|u_{k}\right|^{2}|L(k)|^{2}=\sum_{|k| \leqq j}\left(1+|k|^{2}\right)^{s}\left|f_{k}\right|^{2}<\infty,
$$

$$
\left\|f-L(D) \varphi_{j}\right\|_{s} \rightarrow 0 \quad \text { für } j \rightarrow \infty .
$$

Die Relationen (2.8) und (2.9) besagen $L_{s}^{\sim} u=f$.

C. Ist $u \in D\left(L_{s}^{*}\right)$ und $f:=L_{s}^{*} u$, so ist

$$
(u, L(D) \varphi)_{s}=(f, \varphi)_{s} \text { für alle } \varphi \in S,
$$


woraus ähnlich wie in Beweisteil A

$$
\overline{L(k)} u_{k}=f_{k} \quad \text { für alle } k \in Z^{n}
$$

folgt, und dies ergibt (2.5).

D. Da jedes Element $u \in D\left(L_{s}^{\sim}\right)$ eine starke und somit auch eine schwache $H_{\pi}^{s}$-Lösung der zugeordneten Differentialgleichung

$$
L(D) u=\tilde{L_{s}} u
$$

ist, gilt (2.6) für $u \in D\left(L_{s}^{\sim}\right)$. Umgekehrt ist $u \in H_{\pi}^{s}$ mit Eigenschaft (2.6) nach B eine starke $H_{\pi}^{s}$-Lösung der Differentialgleichung $L(D) u=f$ mit $f:=\left\{u_{k} L(k)\right\}_{k \in Z^{n}}$, und somit gilt $u \in D\left(L_{s}^{\sim}\right)$.

E. Für $u=\left\{u_{k}\right\}_{k \in Z^{n}} \in N\left(L_{s}^{\sim}\right)$ ist nach (2.4)

$$
L(k) u_{k}=0 \text { für alle } k \in \boldsymbol{Z}^{n},
$$

also gilt $u_{k}=0$ für $L(k) \neq 0$. Die Umkehrung zeigt man analog.

F. Ist $f=\left\{f_{k}\right\}_{k \in Z^{n}} \in H_{\pi}^{s}$ und wird $u=\left\{u_{k}\right\}_{k \in Z^{n}} \in H_{\pi}^{s}$ durch (2.7) definiert, so ist

$$
\sum_{k \in Z^{n}}\left(1+|k|^{2}\right)^{s}\left|u_{k}\right|^{2}|L(k)|^{2}=\sum_{k \in Z^{n}}\left(1+|k|^{2}\right)^{s}\left|f_{k}\right|^{2}<\infty
$$

und folglich $u \in D\left(L_{s}^{\sim}\right)$ mit $L_{s}^{\sim} u=f \in R\left(L_{s}^{\sim}\right)$. Aus diesem geht auch die Umkehrung hervor.

2.2. Für koerzitive Differentialoperatoren mit konstanten Koeffizienten gilt

Satz 2.2. Der Differentialoperator (2.1) mit konstanten Koeffizienten $a_{\sigma} \in \boldsymbol{C}$ sei t-koerzitiv $(t \in \boldsymbol{R}, t>0)$. Für $s \in \boldsymbol{R}$ ist

$$
D\left(L_{s}^{\sim}\right) \subset H_{\pi}^{s+t},
$$

und es gilt die Fredholmsche Alternative

$$
\left\{\begin{array}{l}
\operatorname{dim} N\left(L_{s}^{\sim}\right)=\operatorname{dim} N\left(L_{s}^{*}\right)<\infty, \\
R\left(L_{s}^{\sim}\right)=N\left(L_{s}^{*}\right)^{\perp}, \quad R\left(L_{s}^{*}\right)=N\left(L_{s}^{\sim}\right)^{\perp} .
\end{array}\right.
$$

Überdies ist $N\left(L_{s}^{*}\right)=N\left(L_{s}^{\sim}\right)=N\left(L^{\sim}\right)=N(L) \subset S$ für alle $s \in \boldsymbol{R}$.

Ist speziell $L(D)$ stark $t$-koerzitiv, so gibt es ein $\lambda_{0} \in \boldsymbol{R}, \lambda_{0} \geqq 0$ derart, daß mit jedem $s \in \boldsymbol{R}$ und für alle $\lambda \geqq \lambda_{0}$ die Abbildung $L_{s}^{\sim}+\lambda I: D\left(L_{s}^{\sim}\right) \rightarrow H_{\pi}^{s}$ bijektiv und folglich die inverse Abbildung $\left(L_{s}^{\sim}+\lambda I\right)^{-1}: H_{\pi}^{s} \rightarrow D\left(L_{s}^{\sim}\right)$ stetig ist.

Beweis. A. Aus (1.2a) folgt mit $k \in Z^{n}$ und mit der durch

$$
\psi(x):=(2 \pi)^{-n / 2} e^{i(k, x)}
$$

erklärten Funktion $\psi \in S$ die Ungleichung

und daher

$$
|L(k)| \geqq C_{1}\left(1+|k|^{2}\right)^{t / 2}-C_{2}
$$

$$
|L(k)|^{2} \geqq \frac{C_{1}^{2}}{2}\left(1+|k|^{2}\right)^{t}-C_{2}^{2}
$$


Sei $\varphi=\left\{\varphi_{k}\right\}_{k \in Z^{n}} \in S$. Wir multiplizieren Ungleichung (2.12) mit $\left(1+|k|^{2}\right)^{s}\left|\varphi_{k}\right|^{2}$ und summieren über $k \in Z^{n}$. Somit folgt

$$
\|L(D) \varphi\|_{s}^{2} \geqq \frac{C_{1}^{2}}{2}\|\varphi\|_{s+t}^{2}-C_{2}^{2}\|\varphi\|_{s}^{2} \quad \text { für alle } \varphi \in S .
$$

Hieraus erhält man durch Abschließung die analoge Ungleichung für $L_{s}^{\tilde{s}}$, und daraus ergibt sich (2.10).

B. Gemäß Satz 2.1.C ist $N\left(L_{s}^{\sim}\right)=N\left(L_{s}^{*}\right)$. Nach Satz 2.1.E gilt $u=\left\{u_{k}\right\}_{k \in Z^{n}} \in N\left(L_{s}^{\sim}\right)$ genau dann, wenn $u_{k}=0$ für $L(k) \neq 0$ ist. Wegen der $t$-Koerzitivität von $L(D)$ ist nach Satz $1.2|L(k)|>0$ für $|k| \geqq R$. Für $u \in N\left(L_{s}^{\sim}\right)$ gilt folglich $u_{k}=0$ für $|k| \geqq R$; daher ergibt sich $N\left(L_{s}^{\sim}\right)=N\left(L^{\sim}\right)=N(L) \subset S$ und $\operatorname{dim} N\left(L_{s}^{\sim}\right)<\infty$.

Es seien $\left\{f^{(j)}\right\}_{j \in N} \subset R\left(L_{s}^{\sim}\right)$ und $f \in H_{\pi}^{s}$ mit $\left\|f-f^{(j)}\right\|_{s} \rightarrow 0$ für $j \rightarrow \infty$. Zu $f^{(j)}=\left\{f_{k}^{(j)}\right\}_{k \in Z^{n}}$ sei $u^{(j)}=\left\{u_{k}^{(j)}\right\}_{k \in Z^{n}}$ gemäß Satz 2.1.F durch

$$
u_{k}^{(j)}:= \begin{cases}f^{(j)} / L(k) & \text { für } L(k) \neq 0 \\ 0 & \text { für } L(k)=0\end{cases}
$$

erklärt. Dann ist $L_{s}^{\sim} u^{(j)}=f^{(j)}$, und mit

$$
M:=\max \left\{|L(k)|^{-1} \mid k \in Z^{n} \text { mit }|k| \leqq R, L(k) \neq 0\right\}
$$

folgert man

$$
\begin{aligned}
& \left\|u^{(j)}-u^{(l)}\right\|_{s}^{2} \\
& \leqq \sum_{|k|>R}\left(1+|k|^{2}\right)^{s} \frac{\left|f_{k}^{(j)}-f_{k}^{(l)}\right|^{2}}{E^{2}|k|^{2 t}}+\sum_{|k| \leqq R}\left(1+|k|^{2}\right)^{s} M^{2}\left|f_{k}^{(j)}-f_{k}^{(l)}\right|^{2} \\
& \leqq\left(\frac{1}{E^{2} R^{2 t}}+M^{2}\right)\left\|f^{(j)}-f^{(l)}\right\|_{s}^{2} \rightarrow 0 .
\end{aligned}
$$

Also gibt es ein $u \in H_{\pi}^{s}$ mit $\left\|u-u^{(j)}\right\|_{s} \rightarrow 0$. Da $L_{s}^{\sim}$ abgeschlossen ist, gilt $u \in D\left(L_{s}^{\tilde{s}}\right)$ und $L_{s}^{\sim} u=f$, und $R\left(L_{s}^{\sim}\right)$ ist abgeschlossen.

C. Ähnlich wie in Beweisteil A zeigt man für einen stark $t$-koerzitiven Differentialoperator $L(D)$ mittels $(1.2 \mathrm{~b})$

$$
\|(L(D)+\lambda) \varphi\|_{s} \geqq C_{1}\|\varphi\|_{s+t} \quad \text { für alle } \varphi \in S
$$

und für alle $\lambda \geqq \lambda_{0}:=C_{2}$. Hieraus folgt aber $N\left(L_{s}^{\sim}+\lambda I\right)=\{0\}$ für alle $\lambda \geqq \lambda_{0}$, und daher ist $R\left(L_{s}^{\tilde{s}}+\lambda I\right)=H_{\pi}^{s}$.

2.3. Wir wollen jetzt noch klären, welche von den in Satz 2.2 angegebenen Abbildungseigenschaften (2.10), (2.11) bereits die $t$-Koerzitivität des Differentialoperators $L(D)$ charakterisieren. Man zeigt leicht (unter Benutzung von Satz 2.1), daß für die Abschließung $L_{s}^{\sim}$ von $L_{s}$ die Fredholmsche Alternative (2.11) genau dann gilt, wenn es zwei Konstanten $E>0$ und $R \geqq 0$ derart gibt, daß $|L(k)| \geqq E$ für alle $k \in Z^{n}$ mit $|k| \geqq R$ ist. Die $t$-koerzitiven Differentialoperatoren sind jedoch 
schon durch die in Eigenschaft (2.10) von $D\left(L_{s}^{\sim}\right)$ enthaltene Regularitätsaussage gekennzeichnet.

Satz 2.3. Sei $L(D)$ der Differentialoperator (2.1) mit konstanten Koeffizienten $a_{\sigma} \in \boldsymbol{C}$. Mit $s \in \boldsymbol{R}$ und mit $t \in \boldsymbol{R}, t>0$ gelte

$$
D\left(L_{s}^{\sim}\right) \subset H_{\pi}^{s+t} .
$$

Dann gibt es zwei Konstanten $E>0$ und $R \geqq 0$ derart, daß

$$
|L(k)| \geqq E\left(1+|k|^{2}\right)^{t / 2} \quad \text { für alle } k \in \boldsymbol{Z}^{n} \text { mit }|k| \geqq R .
$$

Beweis. Mit der Graphennorm

$$
\|u\|_{G}:=\|u\|_{s}+\left\|L_{s}^{\sim} u\right\|_{s}
$$

ist $\left(D\left(L_{s}^{\tilde{s}}\right),\|\cdot\|_{G}\right)$ ein Banachraum.

Wir zeigen, daß der durch

$$
(T u)_{k}:=\left(1+|k|^{2}\right)^{t / 2} u_{k} \quad \text { für } k \in \boldsymbol{Z}^{n}
$$

erklärte lineare Operator

abgeschlossen ist:

$$
T:\left(D\left(L_{s}^{\sim}\right),\|\cdot\|_{G}\right) \rightarrow H_{\pi}^{s}
$$

Seien $\left\{u^{(j)}\right\}_{j \in N} \subset D\left(L_{s}^{\sim}\right), u^{(j)}=\left\{u_{k}^{(j)}\right\}_{k \in Z^{n}}, u=\left\{u_{k}\right\}_{k \in Z^{n}} \in D\left(L_{s}^{\sim}\right), g=\left\{g_{k}\right\}_{k \in Z^{n}} \in H_{\pi}^{s}$, und es gelte

$$
\left\|u-u^{(j)}\right\|_{G} \rightarrow 0,\left\|g-T u^{(j)}\right\|_{s} \rightarrow 0 \text { für } j \rightarrow \infty .
$$

Dann gilt für jedes $k \in \boldsymbol{Z}^{n}$ und für $j \rightarrow \infty$

und

$$
\left(1+|k|^{2}\right)^{t / 2} u_{k}^{(j)} \rightarrow\left(1+|k|^{2}\right)^{t / 2} u_{k}
$$

Also ist

$$
\left(1+|k|^{2}\right)^{t / 2} u_{k}^{(j)} \rightarrow g_{k} .
$$

und daher

$$
\left(1+|k|^{2}\right)^{t / 2} u_{k}=g_{k}
$$

$$
\sum_{k \in Z^{n}}\left(1+|k|^{2}\right)^{s+t}\left|u_{k}\right|^{2}=\sum_{k \in Z^{n}}\left(1+|k|^{2}\right)^{s}\left|g_{k}\right|^{2}<\infty .
$$

Folglich ergibt sich $T u=g$, und $T$ ist abgeschlossen.

Nach dem Satz vom abgeschlossenen Graphen ist $T$ stetig. Also existiert ein $\gamma>0$ derart, da $\beta$

gilt. Dies besagt aber

$$
\|T \varphi\|_{s} \leqq \gamma\|\varphi\|_{G} \quad \text { für alle } \varphi \in S
$$

$$
\|\varphi\|_{s+t} \leqq \gamma\left(\left\|L_{s}^{\sim} \varphi\right\|_{s}+\|\varphi\|_{s}\right) \quad \text { für alle } \varphi \in S,
$$

woraus die $t$-Koerzitivität des Differentialoperators $L(D)$ folgt. 


\section{Der Fall variabler Koeffizienten}

3.1. Wir wollen nun die bisher erzielten Resultate auf gewisse Differentialoperatoren mit variablen Koeffizienten erweitern. Hierzu führen wir zuerst eine Halbordnung in der Menge der Polynome ein (diese Halbordnung ist eine für unsere Zwecke angemessene Modifikation derjenigen von Hörmander [6], S. 70-73):

Definition 3.1. Seien $P, Q$ komplexwertige Polynome der Veränderlichen $\xi=\left(\xi_{1}, \ldots, \xi_{n}\right) \in \boldsymbol{R}^{n}$. Wir nennen $P$ schwächer als $Q, P<Q$, falls ein $C \in \boldsymbol{R}, C \geqq 0$ so existiert, daß $|P(k)| \leqq C|Q(k)|$ für alle $k \in Z^{n}$ gilt.

Satz 3.2. Man nehme an, daß

$$
L^{0}(D):=\sum_{|\sigma| \leqq \boldsymbol{r}} a_{\sigma}^{0} D^{\sigma}
$$

ein $t$-koerzitiver $(t \in \boldsymbol{R}, t>0)$ Differentialoperator mit konstanten Koeffizienten $a_{\sigma}^{0} \in \boldsymbol{C}$ ist. Dann gilt:

A. Zu jedem $\alpha \in \boldsymbol{R}, 0<\alpha<1$ gibt es ein $\varepsilon>0$ derart, daß für alle Differentialoperatoren

$$
L(x, D):=\sum_{|\sigma| \leqq r} a_{\sigma}(x) D^{\sigma}
$$

mit Koeffizienten $a_{\sigma} \in C_{\pi}^{0}$ sowie mit

$$
L(x, .)<L^{0} \quad \text { für alle } x \in \bar{W}
$$

und

$$
\max _{x \in \bar{W}} \sum_{|\sigma| \leqq r}\left|a_{\sigma}(x)-a_{\sigma}^{0}\right| \leqq \varepsilon
$$

die Ungleichung

$$
\left\|L(., D) \varphi-L^{0}(D) \varphi\right\| \leqq \alpha\left\|L^{0}(D) \varphi\right\| \quad \text { für alle } \varphi \in S
$$

gilt.

B. Sei $s \in \boldsymbol{R}$. Es gibt zu jedem $\alpha \in \boldsymbol{R}, 0<\alpha<1$ ein $\varepsilon>0$ derart, daß jeder Differentialoperator (3.2) mit Koeffizienten

$$
a_{\sigma} \in H_{\pi}^{|s-1|+1+x+n / 2},
$$

wobei $x \in \boldsymbol{R}, \quad x>0$ ist, sowie mit den Eigenschaften (3.3) und (3.4)

$$
\left\|L(., D) \varphi-L^{0}(D) \varphi\right\|_{s} \leqq \alpha\left\|L^{0}(D) \varphi\right\|_{s}+K\left\|L^{0}(D) \varphi\right\|_{s-1} \text { für alle } \varphi \in S
$$

mit einer von $s$ und von den Koeffizienten $a_{\sigma}$ abhängigen Konstanten $K \geqq 0$ genügt.

Beweis. A. Sei $\Pi_{r}$ der (endlichdimensionale) lineare Raum aller Polynome $P$ der Veränderlichen $\xi \in R^{n}$ (mit komplexen Koeffizienten) vom Grade $\leqq r$. Durch

$$
\|P\|:=\sup _{k \in Z^{\boldsymbol{n}}} \frac{|P(k)|}{1+|k|^{r}}
$$


wird auf $\Pi_{r}$ eine Norm definiert (aus $\|P\|=0$ folgt $P(k)=0$ für alle $k \in Z^{n}$, also ist $P(\xi)=0$ für alle $\left.\xi \in \boldsymbol{R}^{n}\right)$.

Sei $\Lambda\left(L^{0}\right) \subset \Pi_{r}$ der lineare Teilraum der Polynome $P$, die schwächer als $L^{0}$ sind. Sei $Q_{1}, \ldots, Q_{N}$ eine Basis in $\Lambda\left(L^{0}\right)$. Auf $\Lambda\left(L^{0}\right)$ wird durch

$$
\|P\| \|:=\sum_{j=1}^{N}\left|d_{j}\right| \quad \text { für } \quad P:=\sum_{j=1}^{N} d_{j} Q_{j}
$$

auch eine zweite Norm erklärt. Da $\Lambda\left(L^{0}\right)$ endlichdimensional ist, sind die Normen $\|$. II und $\|$.$\| auf \Lambda\left(L^{0}\right)$ einander äquivalent. Insbesondere gibt es eine Konstante $\beta>0$ derart, da $\beta$

$$
\||| P\| \leqq \beta\|P\| \quad \text { für alle } P \in \Lambda\left(L^{0}\right)
$$

gilt.

Wegen $a_{\sigma} \in C_{\pi}^{0}$ folgt für

$$
L(x, .)=: \sum_{j=1}^{N} c_{j}(x) Q_{j}(.) \in \Lambda\left(L^{0}\right)
$$

aus (3.7), daß auch $c_{j} \in C_{\pi}^{0}(j=1, \ldots, N)$ sind. Gilt zusätzlich $a_{\sigma} \in C_{\pi}^{l}$ mit einem $l \in N$, so sind $c_{j} \in C_{\pi}^{l}$.

Ist

$$
L^{0}(.)=: \sum_{j=1}^{N} c_{j}^{0} Q_{j}(.)
$$

so folgt für jedes $x \in \bar{W}$ und für jedes $j=1, \ldots, N$

$$
\begin{aligned}
\left|c_{j}(x)-c_{j}^{0}\right| & \leqq|| L(x, .)-L^{0} \mid \| \\
& \leqq \beta\left\|L(x, .)-L^{0}\right\| \\
& =\beta \sup _{k \in Z^{n}} \frac{\left|\sum_{|\sigma| \leqq r}\left(a_{\sigma}(x)-a_{\sigma}^{0}\right) k^{\sigma}\right|}{1+|k|^{r}} \\
& \leqq \beta \sum_{|\sigma| \leqq r}\left|a_{\sigma}(x)-a_{\sigma}^{0}\right| \\
& \leqq \beta \max _{x \in \bar{W}} \sum_{|\sigma| \leqq r}\left|a_{\sigma}(x)-a_{\sigma}^{0}\right| .
\end{aligned}
$$

Daher ist

$$
\sum_{j=1}^{N} \max _{x \in \bar{W}}\left|c_{j}(x)-c_{j}^{0}\right| \leqq N \beta \max _{x \in \bar{W}} \sum_{|\sigma| \leqq r}\left|a_{\sigma}(x)-a_{\sigma}^{0}\right|
$$

Da die Polynome $Q_{j}$ schwächer als $L^{0}$ sind, gibt es zu jedem $j=1, \ldots, N$ ein $\eta_{j} \geqq 0$ derart, daß $\left|Q_{j}(k)\right| \leqq \eta_{j}\left|L^{0}(k)\right|$ für alle $k \in \boldsymbol{Z}^{n}$ gilt. Mit $\eta:=\max _{j=1, \ldots, N} \eta_{j}$ 
wird wegen (3.9) für alle $\varphi \in S$

$$
\begin{aligned}
& \left\|L(., D) \varphi-L^{0}(D) \varphi\right\| \\
& =\left\|\sum_{j=1}^{N}\left(c_{j}(.)-c_{j}^{0}\right) Q_{j}(D)\right\| \\
& \leqq \sum_{j=1}^{N}\left\|Q_{j}(D) \varphi\right\| \max _{x \in \bar{W}}\left|c_{j}(x)-c_{j}^{0}\right| \\
& \leqq \sum_{j=1}^{N} \eta_{j}\left\|L^{0}(D) \varphi\right\| \max _{x \in \bar{W}}\left|c_{j}(x)-c_{j}^{0}\right| \\
& \leqq N \beta \eta\left\|L^{0}(D) \varphi\right\| \max _{x \in \bar{W}} \sum_{|\sigma| \leqq r}\left|a_{\sigma}(x)-a_{\sigma}^{0}\right| .
\end{aligned}
$$

Mit $\varepsilon:=\alpha /(N \beta \eta)$ folgt hieraus Ungleichung (3.5).

B. Sei $s \in \boldsymbol{R}$. Für die Behandlung des Ausdruckes

$$
\left\|d^{(j)} Q_{j}(D) \varphi\right\|_{s}
$$

mit $d^{(j)}(x):=c_{j}(x)-c_{j}^{0}$ brauchen wir eine einem Resultat von Lions und Magenes [9], Lemma 7.1, S. 31, analoge Abschätzung für Fourierreihen. Es seien $d^{(j)}=:\left\{d_{k}^{(j)}\right\}_{k \in Z^{n}}$, $\varphi \in S$ und $Q_{j}(D) \varphi=: \psi^{j}=:\left\{\psi_{k}^{(j)}\right\}_{k \in Z^{n}} \in S$; im folgenden lassen wir zur Vereinfachung der Schreibweise den oberen Index ${ }^{(j)}$ weg. Der Fourierkoeffizient $(d \psi)_{k}$ von $d \psi$ ist dann durch

gegeben. Daher wird

$$
(d \psi)_{k}=\sum_{l \in Z^{n}} d_{l} \psi_{k-l}
$$

$$
\|d \psi\|_{s}=\left(\sum_{k \in Z^{n}}\left(1+|k|^{2}\right)^{s}\left(\sum_{l \in Z^{n}} d_{l} \psi_{k-l}\right)^{2}\right)^{1 / 2} \leqq I_{1}+I_{2}
$$

mit

$$
\begin{aligned}
& I_{1}:=\left(\sum_{k \in Z^{n}}\left(\sum_{l \in Z^{n}}\left(1+|k-l|^{2}\right)^{s / 2} d_{l} \psi_{k-l}\right)^{2}\right)^{1 / 2}, \\
& I_{2}:=\left(\sum_{k \in Z^{n}}\left(\sum_{l \in Z^{n}}\left(\left(1+|k|^{2}\right)^{s / 2}-\left(1+|k-l|^{2}\right)^{s / 2}\right) d_{l} \psi_{k-l}\right)^{2}\right)^{1 / 2} .
\end{aligned}
$$

Mit $g_{k}:=\left(1+|k|^{2}\right)^{s / 2} \psi_{k}$ ist $g:=\left\{g_{k}\right\}_{k \in Z^{n}} \in S$ und

$$
\begin{aligned}
I_{1}^{2} & =\sum_{k \in Z^{n}}\left(\sum_{l \in Z^{n}} d_{l} g_{k-l}\right)^{2}=\|d g\|^{2} \\
& \leqq\left(\max _{x \in \bar{W}}|d(x)|\right)^{2}\|g\|^{2} \\
& =\left(\max _{x \in \bar{W}}|d(x)|\right)^{2}\|\psi\|_{s}^{2} .
\end{aligned}
$$


Nach dem Mittelwertsatz wird für $x, y \in \boldsymbol{R}^{n}$ mit einem $z:=\tau x+(1-\tau)(x-y)=$ $x-y+\tau y, \quad 0<\tau<1$,

\section{Wegen}

$$
\begin{aligned}
& \left|\left(1+|x|^{2}\right)^{s / 2}-\left(1+|x-y|^{2}\right)^{s / 2}\right| \\
& =|s|\left|\sum_{j=1}^{n}\left(1+|z|^{2}\right)^{(s-2) / 2} z_{j} y_{j}\right| \\
& \leqq|s|\left(1+|z|^{2}\right)^{(s-2) / 2}|z||y| \\
& \leqq|s|\left(1+|z|^{2}\right)^{(s-1) / 2}|y| \text {. }
\end{aligned}
$$

ist für $s-1 \geqq 0$

$$
\left(1+|z|^{2}\right)^{1 / 2} \leqq 1+|z| \leqq 2^{1 / 2}\left(1+|z|^{2}\right)^{1 / 2}
$$

und für $s-1<0$

$$
\begin{aligned}
& \left(1+|z|^{2}\right)^{(s-1) / 2} \leqq(1+|z|)^{s-1} \\
& \leqq(1+|x-y+\tau y|)^{s-1} \\
& \leqq(1+|x-y|)^{s-1}(1+|y|)^{s-1} \\
& \leqq 2^{(s-1) / 2}\left(1+|x-y|^{2}\right)^{(s-1) / 2}(1+|y|)^{s-1}
\end{aligned}
$$

$$
\begin{aligned}
& \left(1+|z|^{2}\right)^{(s-1) / 2} \leqq 2^{(1-s) / 2}(1+|z|)^{s-1} \\
& =2^{(1-s) / 2}\left(\frac{1+|x-y|}{(1+|x-y|)(1+|x-y+\tau y|)}\right)^{1-s} \\
& \leqq 2^{(1-s) / 2}\left(\frac{1+|x-y+\tau y|+|y|}{(1+|x-y|)(1+|x-y+\tau y|)}\right)^{1-s} \\
& \leqq 2^{(1-s) / 2}(1+|x-y|)^{s-1}(1+|y|)^{|s-1|} \\
& \leqq 2^{|s-1| / 2}\left(1+|x-y|^{2}\right)^{(s-1) / 2}(1+|y|)^{|s-1|} .
\end{aligned}
$$

Somit folgt für $k, l \in \boldsymbol{Z}^{n}$

$$
\left|\left(1+|k|^{2}\right)^{s / 2}-\left(1+|k-l|^{2}\right)^{s / 2}\right|
$$

Daher ergibt sich

$$
\leqq|s| 2^{|s-1|}\left(1+|k-l|^{2}\right)^{(s-1) / 2}\left(1+|l|^{2}\right)^{(|s-1|+1) / 2} \text {. }
$$

$$
\begin{aligned}
I_{2} & \leqq|s| 2^{|s-1|}\left(\sum_{k \in Z^{n}}\left(\sum_{l \in Z^{n}}\left(1+|k-l|^{2}\right)^{(s-1) / 2}\left|\psi_{k-l}\right|\left(1+|l|^{2}\right)^{(|s-1|+1) / 2}\left|d_{l}\right|\right)^{2}\right)^{1 / 2} \\
& \leqq|s| 2^{|s-1|}\|\psi\|_{s-1} \sum_{l \in Z^{n}}\left(1+|l|^{2}\right)^{(|s-1|+1) / 2}\left|d_{l}\right| .
\end{aligned}
$$

Es gilt

mit

$$
\begin{aligned}
& \sum_{l \in Z^{n}}\left(1+\left.|l|\right|^{2}\right)^{(|s-1|+1) / 2}\left|d_{l}\right| \\
= & \sum_{l \in Z^{n}}\left(1+|l|^{2}\right)^{(|s-1|+1+x+n / 2) / 2}\left|d_{l}\right|\left(1+|l|^{2}\right)^{-(x+n / 2) / 2} \\
\leqq & K_{n}\|d\|_{|s-1|+1+x+n / 2}
\end{aligned}
$$

$$
K_{n}:=\left(\sum_{l \in Z^{n}}\left(1+|l|^{2}\right)^{-(x+n / 2)}\right)^{1 / 2} .
$$


Folglich erhält man

mit

$$
\begin{aligned}
& \left\|L(., D) \varphi-L^{0}(D) \varphi\right\|_{s} \\
& \leqq \sum_{j=1}^{N}\left(\max _{x \in \bar{W}}\left|c_{j}(x)-c_{j}^{0}\right|\right)\left\|Q_{j}(D) \varphi\right\|_{s} \\
& \quad+K_{n, s} \sum_{j=1}^{N}\left\|d^{(j)}\right\|_{|s-1|+1+x+n / 2}\left\|Q_{j}(D) \varphi\right\|_{s-1}
\end{aligned}
$$

$$
K_{n, s}:= \begin{cases}K_{n}|s| 2^{|s-1|} & \text { für } s \neq 0 \\ 0 & \text { für } s=0 .\end{cases}
$$

Ähnlich wie in (3.8) leitet man punktweise Abschätzungen für die Ableitungen von $d^{(j)}$ her, und durch Integration ergibt sich

$$
\sum_{j=1}^{N}\left\|d^{(j)}\right\|_{|s-1|+1+x+n / 2} \leqq \sum_{|\sigma| \leqq r}\left\|b_{\sigma}\right\|_{|s-1|+1+x+n / 2},
$$

wobei $b_{\sigma}(x):=a_{\sigma}(x)-a_{\sigma}^{0}$ ist.

Schließlich zeigt man analog wie in Beweisteil A, daß

gilt. Mit $\varepsilon:=\alpha /(N \beta \eta)$ und

$$
\begin{aligned}
& \left\|L(., D) \varphi-L^{0}(D) \varphi\right\|_{s} \\
& \leqq N \beta \eta\left\|L^{0}(D) \varphi\right\|_{s} \max _{x \in \bar{W}} \sum_{|\sigma| \leqq r}\left|a_{\sigma}(x)-a_{\sigma}^{0}\right| \\
& \quad+K_{n, s} \beta \eta\left\|L^{0}(D) \varphi\right\|_{s-1} \sum_{|\sigma| \leqq r}\left\|b_{\sigma}\right\|_{|s-1|+1+x+n / 2}
\end{aligned}
$$

$$
K:=K_{n, s} \beta \eta \sum_{|\sigma| \leqq r}\left\|b_{\sigma}\right\|_{|s-1|+1+x+n / 2}
$$

folgt hieraus Behauptung (3.6).

3.2. Satz 2.2 läßt sich in der folgenden Form auf den Fall variabler Koeffizienten übertragen:

Satz 3.3. Es sei

$$
L^{0}(D):=\sum_{|\sigma| \leqq r} a_{\sigma}^{0} D^{\sigma}
$$

ein stark $t$-koerzitiver $(t \in \boldsymbol{R}, t>0)$ Differentialoperator mit konstanten Koeffizienten $a_{\sigma}^{0} \in C$. Ferner sei

$$
L(x, D):=\sum_{|\sigma| \leqq r} a_{\sigma}(x) D^{\sigma}
$$

ein Differentialoperator mit Koeffizienten $a_{\sigma} \in H_{\pi}^{s}$ mit $s \in \boldsymbol{R}$.

Man setze voraus, daß mit einem $\alpha \in \boldsymbol{R}, 0<\alpha<1$ und mit einem $K \in \boldsymbol{R}, K \geqq 0$

$$
\left\|L(., D) \varphi-L^{0}(D) \varphi\right\|_{s} \leqq \alpha\left\|L^{0}(D) \varphi\right\|_{s}+K\left\|L^{0}(D) \varphi\right\|_{s-1} \text { für alle } \varphi \in S
$$

gilt (man vergleiche Satz 3.2.B). 
Dann ist der in $H_{\pi}^{s}$ durch

$$
\begin{gathered}
D\left(L_{s}\right):=S \subset H_{\pi}^{s}, \\
L_{s} \varphi:=L(., D) \varphi \quad \text { für } \varphi \in S
\end{gathered}
$$

erklärte lineare Operator $L_{s}: D\left(L_{s}\right) \rightarrow H_{\pi}^{s}$ abschließbar mit der kleinsten abgeschlossenen Fortsetzung $L_{s}^{\sim}:=L_{s}^{* *}$, und es gilt

$$
D\left(L_{s}^{\sim}\right) \subset H_{\pi}^{s+t}
$$

Weiter besteht die Fredholmsche Alternative

$$
\left\{\begin{array}{l}
\operatorname{dim} N\left(L_{s}^{\sim}\right)=\operatorname{dim} N\left(L_{s}^{*}\right)<\infty \\
R\left(L_{s}^{\sim}\right)=N\left(L_{s}^{*}\right)^{\perp}, \quad R\left(L_{s}^{*}\right)=N\left(L_{s}^{\sim}\right)^{\perp} .
\end{array}\right.
$$

Dazu existiert ein $\lambda_{s} \in \boldsymbol{R}, \lambda_{s} \geqq 0$ derart, daß für alle $\lambda_{s} \geqq \lambda_{s}$ die Abbildung $L_{s}+\lambda I$ : $D\left(L_{s}^{\sim}\right) \rightarrow H_{\pi}^{s}$ bijektiv und folglich die inverse Abbildung $\left(L_{s}^{\sim}+\lambda I\right)^{-1}: H_{\pi}^{s} \rightarrow D\left(L_{s}^{\sim}\right)$ stetig ist.

Beweis. A. Die starke $t$-Koerzitivität von $L^{0}(D)$ besagt, daß mit Konstanten $C_{1}>0$ und $C_{2} \geqq 0$ die Ungleichung

$$
\operatorname{Re}\left(L^{0}(D) \psi, \psi\right) \geqq C_{1}\|\psi\|_{t / 2}^{2}-C_{2}\|\psi\|^{2} \quad \text { für alle } \psi \in S
$$

gilt. Mit $k \in Z^{n}$ und

ergibt sich hieraus

$$
\psi(x):=(2 \pi)^{-n / 2} e^{i(k, x)}
$$

$$
\operatorname{Re} L^{0}(k) \geqq C_{1}\left(1+|k|^{2}\right)^{t / 2}-C_{2} \text {. }
$$

Es sei $\varphi=\left\{\varphi_{k}\right\}_{k \in Z^{n}} \in S$. Wir multiplizieren Ungleichung (3.12) mit $\left(1+|k|^{2}\right)^{s}\left|\varphi_{k}\right|^{2}$ und summieren dann über $k \in \boldsymbol{Z}^{n}$. Somit ergibt sich

$$
\operatorname{Re}\left(L^{0}(D) \varphi, \varphi\right)_{s} \geqq C_{1}\|\varphi\|_{s+t / 2}^{2}-C_{2}\|\varphi\|_{s}^{2}
$$

und daher für $\lambda \geqq 0$

$$
2 \lambda \operatorname{Re}\left(L^{0}(D) \varphi, \varphi\right)_{s}+\lambda^{2}\|\varphi\|_{s}^{2} \geqq\left(\lambda^{2}-2 \lambda C_{2}\right)\|\varphi\|_{s}^{2} .
$$

Ist $d \geqq 0$ vorgegeben und $\lambda \geqq\left(d+C_{2}^{2}\right)^{1 / 2}+C_{2}$, so erhält man nach (3.13)

$$
\begin{aligned}
& \left\|\left(L^{0}(D)+\lambda\right) \varphi\right\|_{s}^{2} \\
& =\left\|L^{0}(D) \varphi\right\|_{s}^{2}+2 \lambda \operatorname{Re}\left(L^{0}(D) \varphi, \varphi\right)_{s}+\lambda^{2}\|\varphi\|_{s}^{2} \\
& \leqq\left\|L^{0}(D) \varphi\right\|_{s}^{2}+\left(\lambda^{2}-2 \lambda C_{2}\right)\|\varphi\|_{s}^{2} \\
& \leqq\left\|L^{0}(D) \varphi\right\|_{s}^{2}+d\|\varphi\|_{s}^{2} .
\end{aligned}
$$


Nach Voraussetzung (3.6) folgt wegen (3.14)

$$
\begin{aligned}
& \|(L(., D)+\lambda) \varphi\|_{s} \\
& \geqq\left\|\left(L^{0}(D)+\lambda\right) \varphi\right\|_{s}-\left\|L(., D) \varphi-L^{0}(D) \varphi\right\|_{s} \\
& \geqq(1-\alpha)\left\|\left(L^{0}(D)+\lambda\right) \varphi\right\|_{s}-K\left\|L^{0}(D) \varphi\right\|_{s-1} \\
& =\frac{1-\alpha}{2}\left\|\left(L^{0}(D)+\lambda\right) \varphi\right\|_{s} \\
& \quad+\frac{1-\alpha}{2}\left(\left\|\left(L^{0}(D)+\lambda\right) \varphi\right\|_{s}-\frac{2 K}{1-\alpha}\left\|L^{0}(D) \varphi\right\|_{s-1}\right) .
\end{aligned}
$$

Wir setzen $\delta:=2 K /(1-\alpha)$ und wählen

$$
d:=\delta^{2} \max _{|k| \leqq \delta} \frac{\left|L^{0}(k)\right|^{2}}{1+|k|^{2}} .
$$

Nach (3.14) folgt dann für $\lambda \geqq \lambda_{s}:=\left(d+C_{2}^{2}\right)^{1 / 2}+C_{2}$

und hieraus

$$
\begin{aligned}
& \left\|\left(L^{0}(D)+\lambda\right) \varphi\right\|_{s}^{2}-\delta^{2}\left\|L^{0}(D) \varphi\right\|_{s-1}^{2} \\
& \geqq\left\|L^{0}(D) \varphi\right\|_{s}^{2}+d\|\varphi\|_{s}^{2}-\delta^{2}\left\|L^{0}(D) \varphi\right\|_{s-1}^{2} \\
& \geqq \sum_{k \in Z^{n}}\left(1+|k|^{2}\right)^{s-1}\left(\left(1+|k|^{2}\right) d+\left(1+|k|^{2}-\delta^{2}\right)\left|L^{0}(k)\right|^{2}\right)\left|\varphi_{k}\right|^{2} \\
& \geqq \sum_{|k| \geqq \delta}\left(1+|k|^{2}\right)^{s-1}\left(\left(1+|k|^{2}\right) d-\delta^{2}\left|L^{0}(k)\right|^{2}\right)\left|\varphi_{k}\right|^{2} \geqq 0
\end{aligned}
$$

$$
\frac{1-\alpha}{2}\left\|\left(L^{0}(D)+\lambda\right) \varphi\right\|_{s} \geqq K\left\|L^{0}(D) \varphi\right\|_{s-1}
$$

Aus (3.15) ergibt sich somit für $\lambda \geqq \lambda_{s}$

$$
\|(L(., D)+\lambda) \varphi\|_{s} \geqq \frac{1-\alpha}{2}\left\|\left(L^{0}(D)+\lambda\right) \varphi\right\|_{s} \quad \text { für alle } \varphi \in S .
$$

Sei wieder $\varphi=\left\{\varphi_{k}\right\}_{k \in Z^{n}} \in S$. Falls wir in (3.12) zu beiden Seiten $\lambda \geqq \lambda_{s}$ addieren, die so entstehende Ungleichung mit $\left(1+|k|^{2}\right)^{s+t / 2}\left|\varphi_{k}\right|^{2}$ multiplizieren und dann über $k \in \boldsymbol{Z}^{\boldsymbol{n}}$ summieren, so folgt

$$
\begin{aligned}
& C_{1}\|\varphi\|_{s+t}^{2} \leqq \operatorname{Re} \sum_{k \in Z^{n}}\left(L^{0}(k)+\lambda\right)\left(1+|k|^{2}\right)^{s+t / 2}\left|\varphi_{k}\right|^{2} \\
& \leqq\left(\sum_{k \in Z^{n}}\left|L^{0}(k)+\lambda\right|^{2}\left(1+|k|^{2}\right)^{s}\left|\varphi_{k}\right|^{2}\right)^{1 / 2}\left(\sum_{k \in Z^{n}}\left(1+|k|^{2}\right)^{s+t}\left|\varphi_{k}\right|^{2}\right)^{1 / 2} .
\end{aligned}
$$

Mit $\lambda \geqq \lambda_{s}$ gilt daher

und folglich gemäß (3.17)

$$
C_{1}\|\varphi\|_{s+t} \leqq\left\|\left(L^{0}(D)+\lambda\right) \varphi\right\|_{s}
$$

$$
\|(L(., D)+\lambda) \varphi\|_{s} \geqq C_{1} \frac{1-\alpha}{2}\|\varphi\|_{s+t} \quad \text { für alle } \varphi \in S .
$$


B. Sei $\lambda \geqq \lambda_{s}$. Nach (3.17), (3.6) und (3.14) ist

$$
\begin{aligned}
& \frac{1-\alpha}{2}\left\|\left(L^{0}(D)+\lambda\right) \varphi\right\|_{s} \leqq\|(L(., D)+\lambda) \varphi\|_{s} \\
& \leqq\left\|\left(L^{0}(D)+\lambda\right) \varphi\right\|_{s}+\left\|L(., D) \varphi-L^{0}(D) \varphi\right\|_{s} \\
& \leqq\left\|\left(L^{0}(D)+\lambda\right) \varphi\right\|_{s}+\alpha\left\|L^{0}(D) \varphi\right\|_{s}+K\left\|L^{0}(D) \varphi\right\|_{s-1} \\
& \leqq(1+\alpha+K)\left\|\left(L^{0}(D)+\lambda\right) \varphi\right\|_{s} \quad \text { für alle } \varphi \in S .
\end{aligned}
$$

Da $L_{s}^{0}$ abschließbar ist, folgt aus (3.19) die Abschließbarkeit von $L_{s}$ und auch die Relation $D\left(L_{s}^{\sim}\right)=D\left(L_{s}^{0 \sim}\right)$. Die Abschätzungen (3.18) und (3.19) gelten dann auch für die Abschließungen $L_{s}^{0 \sim}$ und $L_{s}^{\sim}$. Aus (3.18) folgt, daß $D\left(L_{s}^{\sim}\right) \subset$ $H_{\pi}^{s+t}$ gilt und daß $R\left(L_{s}^{\sim}+\lambda I\right)$ abgeschlossen für $\lambda \geqq \lambda_{s}$ ist.

C. Wir werden nun zeigen, da $R\left(L_{s}^{\sim}+\lambda I\right)=H_{\pi}^{s}$ für $\lambda \geqq \lambda_{s}$ ist. Gegeben sei $v \in H_{\pi}^{s}$ mit der Eigenschaft

$$
\left(v,\left(L_{s}^{\tilde{s}}+\lambda\right) w\right)_{s}=0 \quad \text { für alle } w \in D\left(L_{s}^{\tilde{\alpha}}\right)=D\left(L_{s}^{0 \sim}\right) .
$$

Wegen $\lambda \geqq \lambda_{s} \geqq C_{2}$ ist $R\left(L_{s}^{0 \sim}+\lambda I\right)=H_{\pi}^{s}$ (man vergleiche Satz 2.2, Beweisteil C), und es existiert ein $u \in D\left(L_{s}^{0 \sim}\right)$ mit

$$
\left(L_{s}^{0 \sim}+\lambda\right) u=v .
$$

Dann folgt nach (3.20), (3.6), (3.14) und (3.16)

$$
\begin{aligned}
& \|v\|_{s}^{2}=\left(v,\left(L_{s}^{0 \sim}+\lambda\right) u\right)_{s} \\
& =\left(v,\left(L_{s}^{\tilde{\alpha}}+\lambda\right) u\right)_{s}+\left(v,\left(L_{s}^{0 \sim}-L_{s}^{\tilde{s}}\right) u\right)_{s} \\
& =\left(v,\left(L_{s}^{0 \sim}-L_{s}^{\sim}\right) u\right)_{s} \\
& \leqq\|v\|_{s}\left\|\left(L_{s}^{0 \sim}-L_{s}^{\sim}\right) u\right\|_{s} \\
& \leqq\|v\|_{s}\left(\alpha\left\|L_{s}^{0 \sim} u\right\|_{s}+K\left\|L_{s}^{0 \sim} u\right\|_{s-1}\right) \\
& \leqq\|v\|_{s}\left(\alpha\left\|\left(L_{s}^{0 \tilde{}}+\lambda\right) u\right\|_{s}+\frac{1-\alpha}{2}\left\|\left(L_{s}^{0 \tilde{}}+\lambda\right) u\right\|_{s}\right) \\
& =\frac{1+\alpha}{2}\|v\|_{s}^{2},
\end{aligned}
$$

und wegen $\alpha<1$ ist $v=0$. Also gilt

sowie nach (3.18)

$$
\begin{gathered}
R\left(L_{s}^{\tilde{x}}+\lambda I\right)=H_{\pi}^{s}, \\
N\left(L_{s}^{*}+\lambda I\right)=R\left(L_{s}^{\tilde{s}}+\lambda I\right)^{\perp}=\{0\} \\
N\left(L_{s}^{\tilde{\alpha}}+\lambda I\right)=\{0\},
\end{gathered}
$$

und $L_{s}^{\tilde{s}}+\lambda I$ ist ein Fredholm-Operator mit Index Null.

D. Der durch

$$
\begin{gathered}
D\left(T_{s}\right):=H_{\pi}^{s} \\
T_{s} u:=-\lambda u \quad \text { für } u \in D\left(T_{s}\right)
\end{gathered}
$$


erklärte lineare Operator $T_{s}: H_{\pi}^{s} \rightarrow H_{\pi}^{s}$ ist $\left(L_{s}^{\sim}+\lambda I\right)$-kompakt: Sei $\left\{u_{j}\right\}_{j \in N}$ eine Folge aus $D\left(L_{s}^{\sim}\right)$, und es gelte mit einer Konstanten $M>0, M<\infty$

Nach (3.18) ist

$$
\left\|u_{j}\right\|_{s}+\left\|\left(L_{s}^{\tilde{s}}+\lambda\right) u_{j}\right\|_{s} \leqq M \quad \text { für alle } j \in N .
$$

$$
\left\|u_{j}\right\|_{s+t} \leqq M \frac{2}{C_{1}(1-\alpha)} \quad \text { für alle } j \in N .
$$

Wegen der Kompaktheit der Einbettung von $H_{\pi}^{s+t}$ in $H_{\pi}^{s}$ (man vergleiche BersSchechter [2], Lemma 10, S. 169-170, der Beweis läßt sich übertragen, und Eells [3], (D) Theorem, S. 111-112) enthält $\left\{u_{j}\right\}_{j \in N}$ eine in $H_{\pi}^{s}$ konvergente Teilfolge $\left\{u_{j}^{\prime}\right\}_{j \in N}$, also konvergiert $\left\{T_{s} u_{j}^{\prime}\right\}_{j \in N}$ in $H_{\pi}^{s}$.

Da der Index von $L_{s}^{\tilde{s}}+\lambda I$ Null war, ist auch der Index von $L_{s}^{\tilde{s}}=\left(L_{s}^{\tilde{s}}+\lambda I\right)+T_{s}$ Null (man vergleiche Kato [7], S. 238), und daher gilt $\operatorname{dim} N\left(L_{s}^{\sim}\right)=\operatorname{dim} N\left(L_{s}^{*}\right)<\infty$.

3.3. Wir beweisen jetzt einige Regularitätsaussagen:

Satz 3.4. Es seien $t, s, l \in \boldsymbol{R}$ mit $t>0$ und $l<s$. Der Differentialoperator

$$
L^{0}(D):=\sum_{|\sigma| \leqq r} a_{\sigma}^{0} D^{\sigma}
$$

mit konstanten Koeffizienten $a_{\sigma}^{0} \in \boldsymbol{C}$ sei stark t-koerzitiv. Ferner sei

$$
L(x, D):=\sum_{|\sigma| \leqq r} a_{\sigma}(x) D^{\sigma}
$$

ein Differentialoperator mit Koeffizienten $a_{\sigma} \in H_{\pi}^{e}$ mit $\varrho:=\max \{|s|,|l|\}$. Man setze voraus, daß mit einem $\alpha \in \boldsymbol{R}, 0<\alpha<1$ und mit einem $K \in \boldsymbol{R}, K \geqq 0$

$$
\left\|L(., D) \varphi-L^{0}(D) \varphi\right\|_{s} \leqq \alpha\left\|L^{0}(D) \varphi\right\|_{s}+K\left\|L^{0}(D) \varphi\right\|_{s-1} \quad \text { für alle } \varphi \in S
$$

gilt.

Weiter seien $f \in H_{\pi}^{s}$ und $u \in D\left(L_{l}^{\tilde{\nu}}\right)$ derart, daß $L_{l}^{\tilde{l}} u=f$ gilt.

Dann ist $u \in D\left(L_{s}^{\sim}\right) \subset H_{\pi}^{s+t}$ und $L_{s}^{\sim} u=f$.

Beweis. Sei $m \in N$ mit der Eigenschaft $l+(m-1) t<s \leqq l+m t$, und seien $s_{j}:=\min \{l+j t, s\}$ für $j=0, \ldots, m$. Nach Satz 3.3 existiert zu jedem $j$ ein $\lambda_{s_{j}} \geqq 0$ derart, daß die Abbildung $L_{s_{j}}^{\sim}+\lambda I: D\left(L_{s_{j}}^{\tilde{\alpha}}\right) \rightarrow H_{\pi}^{s_{j}}$ mit $D\left(L_{s_{j}}^{\tilde{\alpha}}\right) \subset H_{\pi}^{s_{j}+t}$ für $\lambda \geqq \lambda_{s_{j}}$ bijektiv ist. Es sei $\lambda_{0}:=\max \left\{\lambda_{s_{0}}, \ldots, \lambda_{s_{m}}\right\}$.

Durch Induktion werden wir zeigen, daß $u \in D\left(L_{s_{j}}^{\sim}\right)$ und $\tilde{L}_{s_{j}}^{\tilde{u}} u=f$ für $j=0, \ldots, m$ gilt.

Nach Voraussetzung gilt die Behauptung für $j=0$. Sei also $0<j<m$ (und daher $\left.s_{j}=l+j t<s\right)$ und $u \in D\left(L_{s_{j}}^{\sim}\right)$ mit $\quad L_{s_{j}}^{\sim} u=f$. Wegen $D\left(L_{s_{j}}^{\sim}\right) \subset H_{\pi}^{s_{j}+t} \subset H_{\pi}^{s_{j+1}}$ ist $f+\lambda_{0} u \in H_{\pi}^{s_{j+1} \subset H_{\pi}^{s_{j}}}$ und $\left(L_{s_{j}}^{\tilde{s}_{j}}+\lambda_{0}\right) u=f+\lambda_{0} u$. Es gibt ein $v \in D\left(L_{s_{j+1}}^{\sim}\right)$ mit $\left(L_{s_{j+1}}^{\tilde{y}}+\lambda_{0}\right) v=f+\lambda_{0} u$. Wegen $D\left(L_{s_{j+1}}^{\tilde{1}}\right) \subset D\left(L_{s_{j}}^{\tilde{s}}\right)$ ist auch

$$
\left(L_{s_{j}}^{\sim}+\lambda_{0}\right) v=f+\lambda_{0} u=\left(L_{s_{j}}^{\sim}+\lambda_{0}\right) u \text {, }
$$

also $u=v \in D\left(\tilde{s_{s_{j+1}}}\right)$ und $L_{s_{j+1}}^{\tilde{y}} u=f$. 
Für $j=m$ ist $s_{m}=s$ sowie folglich $D\left(L_{s}^{\sim}\right) \subset H_{\pi}^{s+t}$ und $L_{s}^{\sim} u=f$.

Korollar 3.5. Es seien

$$
L^{0}(D):=\sum_{|\sigma| \leqq r} a_{\sigma}^{0} D^{\sigma}
$$

ein stark $t$-koerzitiver ( $t \in \boldsymbol{R}, t>0)$ Differentialoperator mit konstanten Koeffizienten $a_{\sigma}^{0} \in \boldsymbol{C}$ und

$$
L(x, D):=\sum_{|\sigma| \leqq r} a_{\sigma}(x) D^{\sigma}
$$

ein Differentialoperator mit Koeffizienten $a_{\sigma} \in H_{\pi}^{s} \quad(s \in \boldsymbol{R}, s>0)$.

Man setze voraus, daß mit einem $\alpha \in \boldsymbol{R}, 0<\alpha<1$ und mit einem $K \in \boldsymbol{R}, K \geqq 0$ die Ungleichungen

$$
\left\|L(., D) \varphi-L^{0}(D) \varphi\right\| \leqq \alpha\left\|L^{0}(D) \varphi\right\|
$$

und

$$
\left\|L(., D) \varphi-L^{0}(D) \varphi\right\|_{s} \leqq \alpha\left\|L^{0}(D) \varphi\right\|_{s}+K\left\|L^{0}(D) \varphi\right\|_{s-1}
$$

für alle $\varphi \in S$ gelten.

Dann gilt $N\left(L_{s}^{\sim}\right)=N\left(L_{-s}^{\sim}\right)=N\left(L^{\sim}\right)=N\left(L^{0}\right) \subset S$, wobei $L:=L_{0}$ ist.

Beweis. Wegen $D\left(L_{s}^{\sim}\right) \subset D\left(L_{-s}^{\sim}\right)$ gilt $N\left(L_{s}^{\sim}\right) \subset N\left(L_{-s}^{\sim}\right)$. Nach Satz 3.4 folgt aber $N\left(L_{-s}^{\sim}\right) \subset N\left(L^{\sim}\right) \subset N\left(L_{s}^{\sim}\right)$. Daher hat man $N\left(L_{s}^{\sim}\right)=N\left(L^{\sim}\right)=N\left(L_{-s}^{\sim}\right)$. Aus Voraussetzung (3.5) folgt durch Abschließung

$$
(1-\alpha)\left\|L^{0 \sim} w\right\| \leqq\left\|L^{\sim} w\right\| \leqq(1+\alpha)\left\|L^{0 \sim} w\right\| \quad \text { für alle } w \in D\left(L^{\sim}\right)=D\left(L^{0^{\sim}}\right) .
$$

Folglich ergibt sich $N\left(L^{\sim}\right)=N\left(L^{0^{\sim}}\right)$ und gemäß Satz 2.2 ferner $N\left(L^{0^{\sim}}\right)=N\left(L^{0}\right) \subset S$.

3.4. Wir geben noch eine hinreichende Bedingung dafür an, daß jede schwache $H_{\pi}^{0}$-Lösung der Differentialgleichung $L(., D) u=f$ auch eine starke $H_{\pi}^{0}$-Lösung dieser Differentialgleichung sei:

Satz 3.6. Es sei

$$
L^{0}(D):=\sum_{|\sigma| \leqq r} a_{\sigma}^{0} D^{\sigma}
$$

ein stark t-koerzitiver $(t \in \boldsymbol{R}, t>0)$ Differentialoperator mit konstanten Koeffizienten $a_{\sigma}^{0} \in \boldsymbol{C}$. Ferner sei

$$
L(x, D):=\sum_{|\sigma| \leqq r} a_{\sigma}(x) D^{\sigma}
$$

ein Differentialoperator mit Koeffizienten $a_{\sigma} \in C_{\pi}^{|\sigma|}$ und $L^{\prime}(., D)$ der dem Differentialoperator $L(., D)$ formal adjungierte Differentialoperator.

Man setze voraus, daß mit $\alpha, \alpha^{\prime} \in \boldsymbol{R}, 0<\alpha, \alpha^{\prime}<1$ und mit $K, K^{\prime} \in \boldsymbol{R}, \quad K \geqq 0$, $K^{\prime} \geqq 0$ die Ungleichungen

$$
\begin{gathered}
\left\|L(., D) \varphi-L^{0}(D) \varphi\right\| \leqq \alpha\left\|L^{0}(D) \varphi\right\|+K\left\|L^{0}(D) \varphi\right\|_{-1}, \\
\left\|L^{\prime}(., D) \varphi-L^{0}(D) \varphi\right\| \leqq \alpha^{\prime}\left\|L^{0}(D) \varphi\right\|+K^{\prime}\left\|L^{0}(D) \varphi\right\|_{-1}
\end{gathered}
$$

für alle $\varphi \in S$ gelten. 
Dann ist $L$ wesentlich maximal, $L^{\sim}=L^{\prime *}$. Für $f \in H_{\pi}^{0}$ ist folglich jede schwache $H_{\pi}^{0}$-Lösung $u \in D\left(L^{\prime *}\right)$ der Differentialgleichung $L(., D) u=f$ auch eine starke $H_{\pi}^{0}$-Lösung $u \in D\left(L^{\sim}\right)$ dieser Differentialgleichung.

Beweis. Trivialerweise ist $L^{\sim} \subset L^{\prime *}$.

Weil wegen der Voraussetzung $a_{\sigma} \in C_{\pi}^{|\sigma|}$ die Koeffizienten von $L^{\prime}(., D)$ in $H_{\pi}^{0}$ liegen, existiert gemäß Satz 3.3 (mit $s=0$ ) ein $\lambda^{*} \in \boldsymbol{R}, \lambda^{*} \geqq 0$ derart, daß $R\left(L^{\prime \sim}+\lambda I\right)=$ $R\left(L^{\sim}+\lambda I\right)=H_{\pi}^{0}$ für $\lambda \geqq \lambda^{*}$ ist.

Sei nun $u \in D\left(L^{\prime *}\right)$ und $g:=L^{\prime *} u$. Man wähle eine Zahl $\lambda \geqq \lambda^{*}$. Zu $g+\lambda u$ existiert dann ein $v \in D\left(L^{\sim}\right)$ mit $\left(L^{\sim}+\lambda\right) v=g+\lambda u$. Weil $u \in D\left(L^{\prime *}\right)$ eine schwache $H_{\pi}^{0}$-Lösung der Differentialgleichung $L(., D) u=g$ ist, gilt

$$
\left(\left(L^{\prime}+\lambda\right) \varphi, u\right)=(\varphi, g+\lambda u)=\left(\varphi,\left(L^{\sim}+\lambda\right) v\right)
$$

für alle $\varphi \in S$. Zu $v$ existiert eine Folge $\left\{\psi_{j}\right\}_{j \in N} \subset S$ mit $\left\|v-\psi_{j}\right\| \rightarrow 0$ und $\left\|L^{\sim} v-L \psi_{j}\right\| \rightarrow 0$ für $j \rightarrow \infty$, und es gilt

$$
\begin{aligned}
& \left(\varphi,\left(L^{\sim}+\lambda\right) v\right)=\lim _{j \rightarrow \infty}\left(\varphi,(L+\lambda) \psi_{j}\right) \\
& =\lim _{j \rightarrow \infty}\left(\left(L^{\prime}+\lambda\right) \varphi, \psi_{j}\right)=\left(\left(L^{\prime}+\lambda\right) \varphi, v\right) .
\end{aligned}
$$

Also folgt

und daher auch

$$
\left(\left(L^{\prime}+\lambda\right) \varphi, u-v\right)=0 \quad \text { für alle } \varphi \in S
$$

$$
\left(\left(L^{\prime \sim}+\lambda\right) w, u-v\right)=0 \quad \text { für alle } w \in D\left(L^{\prime \sim}\right) .
$$

Hieraus ergibt sich $u=v$, und somit ist $L^{\sim}=L^{\prime *}$.

Die Verfasser danken Herrn Jouko Tervo für einige Verbesserungsvorschläge.

\section{Literatur}

[1] Agmon, S.: Lectures on elliptic boundary value problems. - Van Nostrand Mathematical Studies 2. D. Van Nostrand Company, Inc., Princeton, New Jersey-TorontoNew York-London, 1965.

[2] Bers, L., und M. Schechter: Elliptic equations. - Partial differential equations [by L. Bers, F. John, M. Schechter]. Lectures in applied mathematics [Proceedings of the summer seminar, Boulder, Colorado, 1957] III. Interscience Publishers, a division of John Wiley \& Sons, Inc., New York-London-Sydney, 1964, 131-299.

[3] Eells, J.: Elliptic operators on manifolds. - Complex analysis and its applications [Lectures presented at an international seminar course at Trieste, 1975] I. International Centre for Theoretical Physics, Trieste. International Atomic Energy Agency, Vienna, 1976, 95-152.

[4] Hildebrandt, S.: Lineare elliptische Differentialgleichungen. - [Vorlesung, Mainz, 1966, als Manuskript vervielfältigt.] Johannes Gutenberg - Universität, Mathematisches Institut, Mainz, 1966. 
[5] HöRMANDER, L.: On the theory of general partial differential operators. - Acta Math. 94, 1955, $161-248$.

[6] HöRMANDER, L.: Linear partial differential operators. - Die Grundlehren der mathematischen Wissenschaften 116. Springer-Verlag, Berlin-Göttingen-Heidelberg, 1963.

[7] Kato, T.: Perturbation theory for linear operators. - Die Grundlehren der mathematischen Wissenschaften 132. Springer-Verlag, Berlin-Heidelberg-New York, 1966.

[8] LAX, P. D.: On Cauchy's problem for hyperbolic equations and the differentiability of solutions of elliptic equations. - Comm. Pure Appl. Math. 8, 1955, 615-633.

[9] Lions, J. L., und E. MAGENES: Non-homogeneous boundary value problems and applications. I. - Die Grundlehren der mathematischen Wissenschaften 181. Springer-Verlag, Berlin-Heidelberg-New York, 1972.

[10] LouhivaAra, I. S., und C. G. Simader: Über nichtelliptische lineare partielle Differentialoperatoren mit konstanten Koeffizienten. - Ann. Acad. Sci. Fenn. Ser. A. I. Math. $513,1972,1-22$.

Universität Jyväskylä

Mathematisches Institut

Sammonkatu 6

SF-40100 Jyväskylä 10

Finnland

Freie Universität Berlin

I. Mathematisches Institut

Hüttenweg 9

D 1000 Berlin 33
Universität Bayreuth

Lehrstuhl III für Mathematik

Opernstraße 22

D 8580 Bayreuth

Bundesrepublik Deutschland

Eingegangen am 10. Mai 1978 